\title{
Marginal bone loss and dental implant failure may be increased in smokers
}

\author{
Abstracted from \\ Moraschini V, Barboza Ed. \\ Success of dental implants in smokers and non-smokers: a systematic review and meta-analysis. \\ Int J Oral Maxillofac Surg 2016; 45: 205-215. doi: 10.1016/j.ijom.2015.08.996. \\ Address for correspondence: Vittorio Moraschini Filho, Department of Periodontology, \\ School of Dentistry, Fluminense Federal University, Rua Mario dos Santos Braga, 30, Centro, \\ Niterói, Rio de Janeiro CEP 24020-140, Brazil. E-mail: vittoriomf@terra.com.br
}

\section{Question: Is there a difference in marginal bone loss or implant failure rates between smokers and non-smokers, depending on the follow-up period?}

Data sources An electronic search was performed in PubMed, Web of Science and the Cochrane Central Register of Controlled Trials up to February 2015. References of included studies were also searched. No language restrictions were applied

Study selection Prospective, retrospective and randomised clinical trials that compared marginal bone loss and failure rates between smokers and non-smokers. Implant failure was considered as total loss of the implant. Studies with patients who had periodontal disease prior to treatment or who had metabolic diseases were excluded. Data extraction and synthesis Two reviewers were involved in the research and screening process and disagreements were resolved by discussion. The quality of the studies was analysed using the Newcastle-Ottawa scale for non-randomised clinical trials. Data extracted from the studies included, when available: follow up period, number of subjects, smoking status, number of implants placed, implant system, implant length and diameter, healing period, antibiotics and mouth-rinse use, marginal bone loss, failure rate and drop-outs. For binary outcomes (implant failure) the estimate of the intervention effect was expressed in the form of an odds ratio (OR) with the confidence interval $(\mathrm{Cl})$ of $95 \%$. For continuous outcomes (marginal bone loss) the average and standard deviation (SD) were used to calculate the standardised mean difference with a $95 \% \mathrm{CI}$. Meta-analysis was performed for studies with similar outcomes, $\mathrm{I}^{2}$ a statistical test was used to express the heterogeneity among the studies. Publication bias was explored as well.

Results A total of 15 observational studies were included in the review. The number of participants ranged from 60 to 1727 and the average age was 52.5 years. The follow-up period ranged from eight to 240 months. The total number of implants placed was 5840 in smokers and 14,683 in non-smokers. The Branemak system, (Noble Biocare AB, Goteborg, Sweden), was the most commonly used implant system. There was a statistically significant difference in marginal bone loss favouring the non-smoking group (SMD $0.49,95 \% \mathrm{Cl} 0.07-0.90)$. There was an increase in marginal bone loss in the maxillae of smokers compared to the mandible (SMD 0.40, 95\% Cl 0.24-0.55) and a statically significant difference in implant failure in favour of the non-smoking group OR 1.96 , 95\% Cl 1.68-2.30.
Conclusions The risk of marginal bone loss and implant failure is increased in smokers compared to non-smokers, however, the results should be interpreted with caution since the data from the review are provided by retrospective and cohort studies.

\section{Commentary}

Smoking in spite of everything is one of the main public health concerns worldwide regardless of the efforts to encourage smoking cessation due to the well known impact of smoking on both general and oral health. As oral health providers we still have to face issues that patients who still smoke may have due to the negative impact in varying degrees on oral health and on the success of some dental procedures. The aim of the review was to evaluate the marginal bone loss and implant failure rates between smokers and non-smokers, which have been investigated in other previous reviews. ${ }^{1-5}$

The review appropriately followed the methodology suggested and adapted the PRISMA statement to conduct the systematic review and searched several databases (three) to look for articles that met their inclusion criteria. Prospective, retrospective and even randomised clinical trials to assess smoking and non-smoking groups were included in the search strategy. As expected the search strategy produced observational studies that were accepted for the review, 15 in total of which 10 were retrospective cohort studies and five were prospective cohort studies. The quality assessment was carried out using a tool for appraisal of non-randomised clinical trials, the Newcastle-Ottawa scale (NOS) and the authors mentioned that with the maximum score of nine on the scale, only two studies scored less than six stars.

The overall results were presented in the narrative section of the published article and were represented in a forest plot figure (metaanalysis). The results analysing marginal bone loss included seven studies in the meta-analysis. The heterogeneity among the studies was very high (the authors calculated $\mathrm{I}^{2}: 98 \%$, (usually less than $40 \%$ is acceptable: the ideal is be close to zero).

The results arrived at a statistically significant result with standard mean difference of 0.49 (95\% CI of 0.07-0.90), with a confidence interval that translates to a modest clinical relevance, for a range of bone loss from $0.07 \mathrm{~mm}$ to $0.9 \mathrm{~mm}$ between smokers and non-smokers, favouring the non-smoking group. Another result examines marginal bone loss and the difference between the mandible and the maxilla. Four studies were analysed for that and the results had a heterogeneity of $0 \%$ and statistically significant results with a standard mean difference of 
0.40 (CI 0.24- 0.50) favouring the mandible. The maxilla may be the area for more bone loss, but again with a precise and narrow confidence interval, translating to a bone loss between $0.24 \mathrm{~mm}$ and $0.5 \mathrm{~mm}$.

For the outcome of failure, the authors divided the results into less than a year, two years, three years, less than five years and more than five years. The overall result of the meta-analysis with a heterogeneity of $23 \%$ presented an OR of 1.96 (CI 1.68-2.30) in favour of the non-smoking group. However, the authors reported on a subgroup analysis for follow-up time which revealed no significant increase in implant failure proportional to the increase in follow-up time.

Previous reviews from 2006 and $2007^{1,2}$ reported similar results with respect to implant failure. The success rate of implants is still considered high and the failure rate is low. The overall implant failure rate reported in studies is more or less $5 \% 3,4,5$ and is dependent upon multiple variables: in other words, the failure rate in smokers may be increased to another $5 \%$ or less (considering the results of the odds ratio of almost two). A fairly small increase in the failure rate should not preclude clinicians using implants in patients who are smokers, and probably a greater discussion should be considered for heavy smokers. The multiple cofounders are difficult to control in observational studies.
Several reviews ${ }^{1-5}$ have examined this same topic with similar or identical outcomes.

We can say that the results show that the implant failure risk is higher in smokers than in non-smokers. However, we should note that while the risk is low for implant failure and marginal bone loss we should interpret the result with caution as the evidence is extracted from observational studies where cofounders are difficult to control and associations can be incorrectly assumed.

Analia Veitz-Keenan New York College of Dentistry, New York, USA

1. Hinode D, Tanabe S, Yokoyama M, Fujisawa K, Yamauchi E, Miyamoto Y. Influence of smoking on osseointegrated implant failure: a meta-analysis. Clin Oral Implants Res 2006; 17: 473-478.

2. Strietzel FP, Reichart PA, Kale A, Kulkarni M, Wegner B, Küchler I. Smoking interferes with the prognosis of dental implant treatment: a systematic review and metaanalysis. / Clin Periodontol 2007; 34: 523-544.

3. Muddugangadhar BC, Amarnath GS, Sonika R, Chheda PS, Garg A. Meta-analysis of Failure and Survival Rate of Implant-supported Single Crowns, Fixed Partial Denture and Implant Tooth-supported Prostheses. J Int Oral Health 2015; 7: 11-17.

4. Moraschini V, Poubel LA, Ferreira VF, Barboza Edos S. Evaluation of survival and success rates of dental implants reported in longitudinal studies with a follow-up period of at least 10years: a systematic review. Int / Oral Maxillofac Surg 2015; 44: 377-388. doi: 10.1016/j.ijom.2014.10.023. Epub 2014 Nov 20.

5. Misch CE, Perel ML, Wang HL, et al. Implant success, survival and failure: the International Congress of Oral Implantologist (ICOI) Pisa Consensus Conference. Implant Dent 2008; 17: 5-15. doi: 10.1097/ID.0b013e3181676059

Evidence-Based Dentistry (2016) 167, 6-7. doi:10.1038/sj.ebd.6401145 